\title{
Will You Talk about God with a Spirituality Chatbot? An Interview Study
}

\author{
Charis Asante-Agyei \\ Syracuse University \\ coasante@syr.edu
}

\author{
Yimin Xiao \\ Syracuse University \\ yxiao39@syr.edu
}

\author{
$\mathrm{Lu}$ Xiao \\ Syracuse University \\ 1xiao04@syr.edu
}

\begin{abstract}
Chatbots are increasingly adopted in our daily activities such as offering customer services and supporting our social activities. Yet, their potential for spiritual purpose is insufficiently explored. Interested in closing this gap, we conducted an interview study with 23 participants comprising 12 Christians, 5 Hindus, 4 Buddhists, 1 Muslim, and 1 Pagan, to probe how people who profess a religion perceive the idea of interacting with a chatbot in a spiritual context. During the interview, we also used a chatbot prototype to engage people in the speculation of a chatbot's roles in religious spaces and the desired functions. Our participants envisioned that spiritual chatbots retrieve religious information for the user. Some welcomed the idea of engaging in a religious conversation with a chatbot while others also expressed concerns of letting chatbots play an active role in religious space.
\end{abstract}

\section{Introduction}

Technologies have been widely used for spiritual purposes. Particularly in religious contexts, the use of video conferencing platforms, streaming websites, and other digital technologies is a growing phenomenon [1]. Church ministers use technologies such as email, World-Wide web, and cellphones to facilitate practices such as worship, pastoral care, research, and reflection [2]. For the lay public and young generation, they use social media, discussion boards, forums, and other digital technologies to access information, socialize with other people, explore religious identities, and form communities $[3,4]$. There are computer programs developed specifically to support people's spiritual activities. For instance, Sun Dial is a mobile application that supports Muslim with their prayers [5]. The Vatican released eRosary, a wearable digital rosary complete with an app that teaches and reminds people to pray. Another technology is the prayer companion, a device that displays information from social networks as prayer topics [6].
This study explores whether and how chatbots can facilitate spiritual activities in religious contexts. Chatbots are online computer programs that engage in conversations with human users [7]. They are increasingly used in various areas, such as business groups' customer service support e.g., [8], personal healthcare assistance [9], tutoring guide for individual learning [7], etc. A chatbot may play roles as a tool, a toy, a friend, or all of them at the same time. Despite their increasing popularity and versatility, there has been limited research and applications of chatbots in spiritual activities. Interested in closing this gap, we interviewed people who profess a religion for their opinions on interacting with a chatbot in spiritual activities. During the interview, we also used a chatbot prototype to engage them in the speculation of a chatbot's role in religious spaces. This exploratory study is our first step towards answering the two following research questions: RQ 1: How do people perceive the use of chatbots for spiritual purposes? RQ2: What preferences do people have for chatbots for spiritual purposes, if any?

We acknowledge the distinction between spirituality and religion [10], though it is beyond the discussion of this study. Currently, this study focuses on spiritual activities in religious contexts. The rest of the paper is organized as follows. We first review the existing work related to the use of chatbot technologies in religious activities. We then describe our research design including the interview questions, the design and development of our chatbot prototype. Next, we present the analysis results and discuss the implications. We conclude with the contributions of the study, its limitations, and suggestions for future research.

\section{Related work}

Advanced digital technologies are becoming more commonplace in everyday life [11]. In religious contexts, there has been research on how communication technology [e.g., 12, 13] and robots [e.g., 14] support people's religious practices, religious community building, and the communication of 
religious life and information. To our best knowledge, there has been relatively little research on the use of chatbots for spiritual purposes in religious contexts. We found three main themes among extant studies: (i) using religious texts to train and evaluate chatbots, (ii) supporting spiritual practices, and (iii) using chatbots for direct spiritual practices. An example of the first theme is [15], which trained two ALICE chatbots using the Qur'an. While both chatbots were trained using Qur'an, one chatbot is monolingual in Arabic and the other one is bilingual in English and Arabic.

A second group of studies develop chatbots to support people with their spiritual practices. For example, one study developed a chatbot that uses Natural Language Preprocessing to find answers based on Islamic law [16]. In another study, [17] reports the development of Esperanza, a chatbot designed to teach people the Bible in Portuguese and Spanish via Facebook Messenger and WhatsApp. The chatbot, Esperanza, was designed with the persona of a disciple (a follower of Jesus) who is friendly, a Theologian, and works 24 hours a day. In the third theme, chatbots are designed to be directly involved in the enactment of spiritual practices. An example is Buddha Bot, an embodied spiritual machine [18]. Buddha Bot is designed to be a digital embodiment of the Buddha, a known religious figure [18].

This study extends existing research in two main ways. First, extant studies have focused on a "topdown" approach that introduces chatbot technologies to the religious communities with preconceived ideas, such as helping participants study religious texts [17] or developing an embodiment of a religious figure [18]. Our interview study takes a "bottom-up" approach, situating participants as "experts of their experience" and engaging them in the field of imagination [19]. Second, existing studies have focused on one religion at a time. However, we include participants from different religious perspectives, enabling us to identify design requirements that are common across multiple religions.

\section{Research methodology}

\subsection{Semi-structured interview}

Given the exploratory nature of this study, we conducted semi-structured interviews of people who are 18 or older and who profess belief in any religion. The first part of the interview focused on understanding the interviewees' background, their religious belief and the religious activities they engage in regularly, as well as their opinions on the use of chatbots in religious activities. It consists of three groups of questions. In the first group, we asked participants to tell us about themselves. Questions covered demographic information such as age group, gender, etc. The second group of questions focused on the participant's religious belief and practices. We asked about the frequency, perceived benefits and challenges associated with those practice. The third group is about their views on chatbots. Questions focused on participants' perceptions of its use in the religious activities they had described.

In the second part of the interview, we presented the screenshots of a chatbot prototype to the interviewees eliciting their feedback. These screenshots are about three scenarios of interacting with the chatbot. We chose these activities because they are common in people's spiritual journey, and building familiarity is important when eliciting feedback using prototypes [20]. We also used a very open question in this part:

I would like to show you some early prototypes of a chatbot. Please offer i) your initial reactions and ii) any use cases you can imagine based on the scenarios.

Nineteen interviewees were recruited through the listservs of the authors' university. In addition, four interviewees were recruited after two authors made a recruitment announcement to their social networks. To address the potential bias in data collection, three of them were interviewed by the researcher who is not in the same social networks as they. Also, two researchers interviewed one participant to address the potential bias as the participant was in one of their social networks. The interview procedure is IRB approved. All interviews took place remotely through Zoom, a video conference application, from Nov. 2020 to Mar. 2021. Each interview lasted between 21 minutes and 65 minutes. Participants gave consent to have the interviews recorded. The interviews were all transcribed and analyzed using a thematic coding approach to allow for emergent themes [21]. Two authors first developed a coding scheme using this approach. Following this coding schema, one of the authors independently annotated all transcripts for two rounds, with two weeks between each round. This annotation process generated 867 coded text segments and the author achieved a $92.73 \%$ consistency percentage between these two rounds of annotation. Focusing on identifying the main benefits and challenges of using chatbot in religious activities perceived by the religious people, we consider that the coding scheme we developed from the data has reflected enough coverage in this exploratory study.

\subsection{Design of chatbot scenarios}

Religious identity is an important theme in digital religion studies and technology offers a place where people explore and negotiate their religious identity [3, $22]$. We envision three scenarios that people encounter in the development of their religious identity and how 
the chatbot plays a role to facilitate the exploration, formation, and/or transformation of their beliefs.

In the first scenario, a user is seeking religious information from the chatbot. As chatbots have been used to provide information and user support [3,22], we envision that users may seek information in a conversation with the chatbot. This scenario helps us explore whether and how chatbots can be a source of religious information, as well as the position of chatbot in people's religious life relative to other sources of religious information - a common inquiry within digital religion studies [3]. In the second scenario, a user engages in a spiritual conversation with the chatbot. Religious socialization mediates the perception and attitudes toward religious beliefs and behaviors [23], and technologies mediate this socialization. For instance, a comparative study in Ghana, Turkey, and Peru finds varying degrees of transformation to selfsocialization for religious purposes among young people [22]. With this scenario, we explore how people perceive the use of a spirituality chatbot for religious socialization and what an interaction like this would mean to them. The third scenario is of the user expressing concerns, confusion, or doubts about their religious beliefs to the chatbot. Religious doubt may be controversial or even prohibited in religious life [24]. We are interested in whether and how the chatbot can provide a conversation space where people feel comfortable to express their concerns and doubt.

\subsection{Development of chatbot prototype}

To implement the scenario design, we built a chatbot prototype using Juji Studio, an online platform that allows users to design, build, and launch the conversation flow and persona of a chatbot, all without coding. It supports a question-answer approach such that a user interacts with the chatbot by scripted inputs and responses based on a collection of question-answer pairs. This approach loses the naturalness of a conversation. However, demoing a chatbot prototype in the interview also helps the interviewees more vividly envision interactions with a chatbot. The prototype also enabled participants to speculate on the desired functions of a chatbot, as well as the concerns of introducing chatbot technologies to the religious space [20]. Therefore, we presented the screenshots of three conversation examples corresponding to the three scenarios. We then probed people's feedback upon viewing them.

Juji uses question-answer pairs to form a knowledge base, from which it retrieves a response to user input. To build these pairs, we leveraged Reddit discussion data based on Bible or Qur'an. We focused on these religious contexts as Christianity and Islam are the two largest religions in the world and have more discussion data in Reddit. To identify the discussion data, we used the tool called Communalytic to find the subreddits that included the keyword "Bible" or "Qur'an". Of these subreddits, we chose the top 15 subreddits for each religion based on the number of posts in a subreddit. We used PRAW, a Python Reddit API Wrapper package, to collect the discussion data. We processed the data into question-answer pairs such that a question was from the title of a discussion or the initial post, and the answer was the first comment in the discussion thread. We uploaded 1110 question-answer pairs from Qur'an discussions and 112 pairs from Bible discussions.

We next designed a conversation flow where the chatbot first greets the user and then provides three conversation options that match the aforementioned scenarios: "I have a question for you", "Just want to talk", and "I am confused about something". Once the user makes a choice, the chatbot acknowledges it and responds based on the uploaded discussion data. Figure 1 shows the screenshot for the first scenario. The user, $y$ in the chat is one of the authors of the paper.

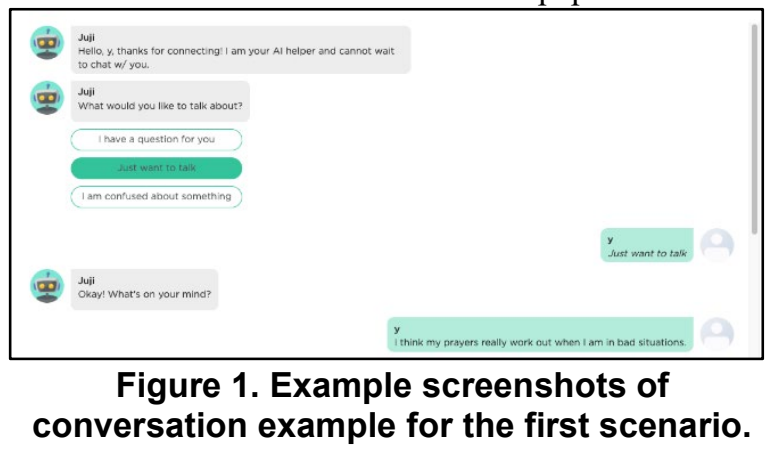

Beyond the three scenarios, our chatbot design also probed the interface design of the chatbot. Past research suggests that human likeness and social characteristics (e.g., conversational intelligence and personification) may play a role in user's interactions with a chatbot $[25$, 26]. In a study of social robots in the religious context [14], the authors introduced two robots with varying appearance and function design to probe user's perception of different robot characteristics. Similarly, we present two chatbot personas in the study: one that has a robot face with the name Juji, and the second with a human name Ava and a female human face. The first persona is used in the first two scenarios, and the second in the third scenario.

\section{Results}

Table 1 shows the major coding families and the number of coded pieces in each family. With this coded content, the authors engaged in collaborative 
sensemaking and validation to achieve a shared understanding of the interview data.

Table 1. Data coding schema

\begin{tabular}{|l|l|}
\hline Description of code families & $\#$ \\
\hline $\begin{array}{l}\text { Chatbot definition and previous chatbot } \\
\text { development or interaction experience }\end{array}$ & 128 \\
\hline $\begin{array}{l}\text { Envisioned chatbot use scenario in } \\
\text { religious contexts }\end{array}$ & 221 \\
\hline $\begin{array}{l}\text { Perceived benefits and enjoyments of/in } \\
\text { religious practices }\end{array}$ & 69 \\
\hline Challenges in religious practices & 59 \\
\hline Religious practices in group settings & 37 \\
\hline Individual religious activities & 103 \\
\hline Religious activities for religious leaders & 5 \\
\hline Human interaction in religious practices & 21 \\
\hline Level of engagement in religious practices & 55 \\
\hline Other aspects of religious practices & 4 \\
\hline $\begin{array}{l}\text { Attitudes towards technology, past } \\
\text { technology use experience, and other } \\
\text { technology use in religious contexts }\end{array}$ & 56 \\
\hline Demographics and religious belief & 113 \\
\hline
\end{tabular}

\subsection{Demographic information}

We interviewed 23 people comprising 12 Christians, 5 Hindus, 4 Buddhists, 1 Muslim, and 1 Pagan. There were 15 females, 7 males, and 1 queer/trans. 17 of the participants are university students aged between 20 to 30.6 participants are between 30 to over 60 years old and are not university students. 17 participants inherited their religion from family or have been influenced by their family's religious beliefs, among which 7 have struggled with their religious belief and explored or switched to alternative beliefs.

15 participants self-reported an average to advanced knowledge of technology. 1 has developed a chatbot, and 4 participants reported that they have certain knowledge of machine learning and AI that are relevant to chatbot technology. The participants shared similar understandings of a chatbot, citing it as a robot, software, or machine driven by algorithms to communicate with a human.

21 participants reported that they have interacted with a chatbot before where the chatbot provides access to answers, customer service, and assistance. In general, participants considered it as a helpful, convenient and time-saving process to get routine customer services when interacting with a chatbot. They also reported that sometimes a chatbot was not intelligent enough to process the input and answer appropriately. They described such experiences as unpleasant and frustrating.

\subsection{Religious practices}

Participants described several of their practices, some of which are individual and others group-based. Practices like praying were daily practices, and others such as going to church, mosque, and temple were weekly. Other practices like giving alms and confession happened when the situation presented itself. Participants associated their spiritual practices with positivity, happiness, peace, and calmness. Religious activities help to structure or center their life, make them feel grounded, and give them a better perspective of life and their relations with the world. The activities also provide them guidance or solace when they face confusion, uncertainty, or difficulties.

Our participants described the challenges they face when engaging in spiritual activities. One common challenge was time constraint and self-discipline. Five participants said they are distant from their families and community, making it hard to engage in religious activities the same way they would do with them and feel connected. The COVID-19 pandemic imposed additional challenges for people to physically participate in religious activities. Additionally, general perception of certain religious practices can also negatively influence people's participation in religious activities. For example, P3 said that religious practices are not as popular among the younger generation and are even associated with negative stigma. Finally, some participants found their own beliefs in conflict with their religion, which makes it hard for them to find a community that they feel included or comfortable with.

\subsection{Envisioning beneficial use for chatbot in religious contexts}

Before presenting them the screen shots of the three chatbot scenarios, we asked the participants whether and how a chatbot could play a role in their spiritual practices. This resulted in three main benefits chatbots can bring to spiritual activities as follows.

\section{Intelligent offering relevant spiritual information}

Majority of the participants acknowledged that a chatbot may help users find information related to their religious belief, e.g., reading materials, mantras, and festivals. For example, P5 envisioned a chatbot helping her find mantras rather than having a book with the mantras, while P7 also thought that chatbots could provide more information concerning festivals for people who, like him, are not as knowledgeable about the religion. P7 went on to say that by using a chatbot in this manner, he could potentially take more interest in his religion. P11 also described an information-retrieval feature he would use: “...what I would probably come 
back to use a chatbot for is if it was more spiritual...So, there are saints in the Catholic Church; and they have feast days where you're supposed to think about the life of that saint and the work that they did. And the lessons you can learn from that can help you in your day to day life. So, if there is a chatbot where I could ask, like, "oh, who's feast day it is?" And then, it gives me a straightforward definition and then I can ask more questions like, "why is it their feast day?" or like, "what were they patrons of?" or like, "which saints were similar to them in terms of what they're the patron of?" Or like "where they're from, originally?" That's just like personally, I would think that it would be really cool from a chatbot."

\section{Reading parts of recited prayers}

Chatbots are envisioned to play a beneficial role in some prayer activities. P2 described a chatbot that could participate in prayers by reading parts of recited prayers. "...I pray the rosary with my family at home. And the rosary, if you are doing it with a group of people, it is one person who leads until they do half of every prayer and then the group responds. And I think that a chatbot could be useful in that sort of way". Speaking of a similar functionality for a chatbot, P13 said, "Well, so during the prayers in church, ...there is like this call and response thing where like the person who's leading the prayers will say one thing, and then the congregation is supposed to respond with this other thing. And I think that a chatbot could like lead a set of prayers in a similar way, if it wanted to...I don't feel like it would be a complete spiritual practice all by itself, but it could be like a neat part of it."

\section{Prompting one to reflect.}

P2 envisioned a chatbot which could help practice gratitude through reflective prayer. "I would say that chatbot can help out with...many different forms of prayer. You can do reflection...you can ask for anything. And the biggest way a chatbot could help is probably in that reflection sense where it can prompt you, 'what are things that happened today?' and maybe help you run throughout your day for you ...".

Participants also envisioned chatbots helping with expressing (repressed) emotions. For instance, P8 commented that "...if they're just looking for, like, a sense of being heard and community, I think a chatbot could fill that". The participant referred a program to further elaborate the idea - "I know that one of the weird functions you can do on a Mac - they have something called "the therapist." So, you can just type things in and it'll just say back like, 'what do you mean by blah, blah, blah.' Or like 'unpack a little bit further'...well, at first, I thought it was silly by having to explain something like going deeper and deeper and deeper into what I meant by a phrase. It actually did like force me to reflect a bit more. if chatbot can be used in that capacity".

\subsection{Concerns of chatbot in religious contexts}

Participants also raised several concerns regarding the use of chatbot in religious activities.

\section{Substituting for human effort}

In general, it is considered an advantage that technologies free humans from mundane efforts like repetition. However, these same efforts can symbolize one's dedication to higher beings in spiritual tasks and be deemed necessary. In this way, chatbots may be substituting a necessary task people want to undertake. For example, P6 described a chatbot that recited prayers as a bad idea, saying, "If they're going to recite it for me, I don't think that's a good idea because I believe that if chatbot is doing that task for me, reciting and reading my prayers, then I don't think I will be able to achieve my ultimate goal that is getting something like inner peace or strength like that because someone else is doing my task there...reciting in place of me is a bad idea." P16 described how this substitution could make Christians lazy to not do what they are meant to do. "I sincerely don't think that it should be the robot's job to share the Gospel...will make the Christians lazy and the fire in their hearts in the sensitivity for the Lord will be declined...".

\section{Affecting human interactions}

P9 spoke about excessive screen time and forming companionship with digital technologies. “...in this day and age, we complain so much about how much screen time we utilize and how that has over time affected our attention span'. Participants also talked about several situations in which they do not think chatbots have a role to play when people seek digested religious knowledge, when people desire human touch, and when people want direct communication with God.

\section{Insufficient domain knowledge}

When envisioning the use of chatbot in religious activities, P3 and P8 acknowledged that it might be helpful for people who are questioning or exploring their faith to interact with a chatbot. P3 thought that a priest would be more knowledgeable than the chatbot so she would direct such questions to the priest. P12 implied that the chatbot may not be in-depth enough, saying- "...in the initial discussions where I don't have a more serious or a more in-depth discussion, I feel like I would be comfortable with the chatbot. But when I generally have to get more insight about a topic, I want 
to go more in-depth about a topic or a discussion, I would like to discuss it with a human".

\section{Unable to provide the human touch}

Participants reported that feelings and connection were a core component of spirituality, which was difficult to simulate with a machine. For example, P7 considered religion a personal thing for which the human touch was important - "Chatbot, No, I don't think so. I can't see any actually fitting...it's kind of personal, a human thing, kind of a lot about the feelings. So, I don't think the chatbot would be playing a role here". P3 also described human touch in this manner, "I don't think it could replace that...just because there's also the human emotion to it ...because yeah, it can bring like a new perspective, but I think it's just the human emotion, like you can take all these facts. But if there's no feeling or emotion behind, it is not really that powerful, in my opinion...".

Human touch in religious activities is also physical. P3 described holding hands when praying, a role she does not think the chatbot could play - "...you hold the hand of the people around you...a chatbot can't necessarily help you with that".

Should not interfere with the communication with God

At least two participants viewed their religion as a relationship between people and God, and thus felt that it is a sacred space where chatbot technologies should not and cannot play. Speaking about relationship with God, P13 described how there should be a space that the chatbot cannot fill - "A lot of spirituality is about having a relationship with God...there has to be space just between you and God". P3 spoke of a similar idea by describing prayer as communication between a person and God - “...it could potentially constitute a prayer, but like I personally wouldn't use it as prayer. I see prayer as more of a conversation or if you're talking to God".

Aside from envisioning a user interacting with a chatbot, two other participants also speculated on the idea of interacting with multiple chatbots for spiritual practices. For example, P14 envisioned having discussions with a group of chatbots, noting that each chatbot could be trained on different scriptures or different gods, which could result in a "healthy discussion". P12 also described being more comfortable chatting with a group of chatbots compared with a single chatbot.

\subsection{Feedback on the three design cases}

As described above, most participants envisioned a chatbot retrieving religious information for them. It is not surprising, then, that their feedback on the first scenario are quite positive. In the first scenario, a user interacts with a chatbot named Juji that has a robot avatar. The user asks the chatbot a question and the chatbot provides information. After reviewing the screenshots of this scenario (Figure 1), P13 commented that it would be desirable for the chatbot to retrieve Christian music- "The entire broad scope of it is possible functionality, but one thing that I might ask you about is, 'where I might find music from a church musical?", P8 and P12 were interested in the kind of questions that people can ask the chatbot and expected the chatbot to intelligently process and synthesize resources from the web rather than performing simple information retrieval tasks.

In the second and third scenario, the user is trying to have a spiritual conversation with the chatbot. Building on this idea, some participants envisioned several situations where they would engage in a conversation with the chatbot: when they feel confused, desperate, or lonely, when they need company to make them feel positive and confident, or when they want to study verses. Participants also shared various concerns and doubts regarding having a spiritual conversation with a chatbot. Some reasons participants had are that the chatbot is not smart enough, it cannot handle an emotional conversation, or it loses the user's attention quickly because it cannot respond well. Others thought that such spiritual conversations should be carried out with a priest or someone who is well established or more in the religious field. P4 commented that "I think a lot of people go to their priests ... because they have already form relation with them just seeing them in Mass every single week, or how often... There's just a little bit more of a relationship bond between that." There was also concern that some users may abuse the chatbot by making trolling conversations, which the chatbot can learn from and adapt.

Participants also offered their design suggestions. Some thought that the chatbot should ask back questions and probe further, while keeping a word limit to the chatbot's response. Other comments on the design revolved around providing options for Q\&As, which can be helpful to navigate the conversation and provide personalized experience and smart responses. Interestingly, besides viewing the chatbot as a conversational partner, P11 also considered that the chat history with the chatbot could be a great place for personal reflection - "...you could kind of go back to prior chats with it and see, like, 'oh, this is how I was feeling on this day'... 'and this is how I was feeling like another day'...kind of like a diary approach.... So I feel like having a chatbot like this would be like a guided meditation in a way where I'm able to talk about my emotions but also have a voice on the other end. You know that's not a human, it's a chatbot...but still". The same interviewee compared this approach with the 
practice of journaling, explaining that it helped him reflect on his emotions and cope with the COVID-19 pandemic. He envisioned that the spiritual chatbot could play a similar role. To him, interacting with this chatbot could be like a "guided meditation" which allows him to talk about his emotions; and the chatbot can check in with the user regularly to keep a record of their feelings and life over time.

In scenario 3, the chatbot is given a female human face and a name, Ava. It also behaves differently from the other chatbots as it uses more empathetic language. In response to this design, two participants thought that the chatbot is ultimately not a real person, so this change does not matter. For instance, P2 said that "I personally wouldn't mind a robot avatar...I know it's a chatbot, and that's okay." P6 thought that Ava appears to be more engaging but "she's just programmed to do that". P4 felt that it was a worse design idea to make the chatbot more human-like - "I maybe treat it, oh, this is a real person. But you don't know him" and "I even want to say more 'No' because I'm vigilant for some of your questions. How can I share my mind with this person? I don't know him.". Other participants however felt that it is important for the chatbot to have the human face and name as it makes the chatbot more human-like and friendly. The language style also makes them feel more comfortable talking to the chatbot. P9 also commented that the robot face in the first two scenarios seemed "creepy".

Broadly defined, empathy is about one's cognitive and emotional responses based on one's perception and understanding of another. There are two types of empathy in the literature: emotional empathy and cognitive empathy [27]. From their responses, participants' concern of a spirituality chatbot being empathetic refers to the cognitive empathy that is about being capable of understanding the other's emotional state and act in a way that is comforting and appropriate in the situation. The participants have expectations that a spirituality chatbot should be cognitively empathetic. For instance, P12 suggested that the chatbot should direct the person in the first example - "How may I help you? Didn't have any questions? That is a good thing to direct a person, what he wants". This reflects the participant's expectation of the chatbot's conversational intelligence. In the second example, the participant made a comment that resonated with several other interviewees - "...I prefer getting a more comfortable response...I get a straightforward respond from a chatbot...This makes me feel like he doesn't take so much interest to my answers or to my opinion".

We also observed a difference in the type of questions people would ask based on the human-like facial features of the chatbot. In the specific case of P1, when asked "if there is a chatbot available. What kind of questions would you want to ask to test it out?", P1 said, "maybe I will ask like "Where will a baby go if a baby just died? Just after he was born but he died suddenly. Will he go to heaven or go to somewhere else?"'. He further explained why he would ask such a question - "I just want to see what he says, I don't know the answer...I think it is a hard one at least for me...But what if he makes a very highly intelligence to response, I will be amazed, right? Or he was like 'oh I don't know' I think that this is not that amazing". However, when the chatbot had the human face in the third scenario, the kind of questions P1 would ask were different - "I may ask something softer...I'm gonna ask which person is the author of which book in Bible... I wouldn't challenge it in that crazy way... Because I think this is another person behind it...I feel a little bit uncomfortable to embarrass it".

\section{Discussion}

\subsection{Design implications}

This exploratory study highlights the versatility of chatbots in spiritual practices, as well as the personal and intimate nature of spiritual and religious practices. Additionally, the wide spectrum of participants' answers calls for further research towards a more critical engagement with advanced intelligence in media and social life $[3,4,11,28]$. In the spirit of our exploratory study, we offer three design implications as follows.

\section{Human-like or not? Let users decide}

We observe different preferences for the chatbot avatar and name, which suggests varying perceptions of an anthropomorphic spirituality chatbot. We interpret these interview results as reflecting participants' trust in the chatbot technology. Trust can be defined as "a psychological state comprising the intention to accept vulnerability based upon positive expectations of the intentions or behavior of another" [29, p. 395]. The literature has shown two perspectives regarding people's trust to AI: Human-Human trust which emphasizes the social relatedness and familiarity, and Human-Machine trust which focuses on the logical and knowledgeable aspect of intelligence [30]. We suggest that as opposed to making design assumptions about the human-likeness of the chatbot's appearance, we make this an option for the users. A list of avatars can be provided ranging from no name and no face to things like human figures, animals, and inanimate objects. The chatbot could also allow users to provide an avatar and name of their choice. Essentially, users will choose the appearance for their chatbot. A user's choice may reflect how they perceive the chatbot as a technology in their 
spiritual life, which is also worth exploring in future research.

Intelligent and data driven or empathetic and understanding? A spirituality chatbot needs both

Apart from human-likeness in the appearance of the chatbot, we also find expectations for the chatbot to have near-human intelligence and empathy. Intelligence is referred to as the chatbot being knowledgeable and capable of providing support to the user based on their "expertise" and data. As presented in the results section, participants focused on the cognitive empathy of spiritual chatbot, illustrating the importance of having such features. It is known that designing for empathy is challenging for offering artificial intelligence (AI), but recent studies provide promising results in this area by leveraging deep learning algorithms to develop the chatbot [e.g., 8, 30]. For a spirituality chatbot, we expect more domain-specific responses from both the intelligence and empathy aspects. We call for more usercentered investigation that probes these requirements and suggestions from the perspectives of people who engage in spiritual activities.

How far can it go? Negotiate the boundary between the chatbot and other agents in the spiritual life

Our interview participants indicate the potential of a chatbot to serve as a guide and companion in various aspects of their spiritual life. While a chatbot, can enable people to access religious information from sources other than their religious leaders, institutions, texts, and practices, its role in people's spiritual life imposes challenges to the status of these traditional religious authorities [4]. According to [31], the authority of traditional leaders can either be complemented or displaced by emerging digital technologies. In the former, traditional religious authorities are reaffirmed by their established status and the relationships they have formed. In the latter, the authority of traditional leaders is weakened when more adherents gain access and more control over religious knowledge previously reserved for only authorities. Furthermore, authorities may no longer serve as gatekeepers to those who do not feel accepted by their religious community because of their contradicting identity or beliefs. It is a design challenge to identify and foster the role of chatbots in the religion space that affords a balanced relationship with other agents. Our interview data suggest two possible approaches. One is to collaborate with the existing leaders and authorities. Some participants reported that they would trust a chatbot more if religious leaders were involved in generating chatbot responses. The other is to focus on helping those who are struggling to find their communities in the existing structure as pointed out by one interviewee.

\subsection{Theoretical and methodological implication}

Besides implications for the design of a spirituality chatbot, our study also contributes to digital religion research. In this interdisciplinary field, scholars study how digital technologies mediate people's perception and presentation of their religious identities, facilitate the development of communities and practices online, and influence the traditional authority associated with religious systems. One theoretical framework in this field is the mediatization theory [31], which frames the meta process by which societal interactions are shaped by a media logic [32]. In this view, media such as television, Internet, and others directly change, intervene, and shape the interaction [32, p. 364]. According to the theory, as media become more pervasive, relations among people and organizations are redefined at the micro-level, along with their meanings at the macro-level [28]. As presented in the results section, chatbots are envisioned to affect how people interact with one other in religious activities. Furthermore, interviewees envisioned how this technology mediates people's interaction with themselves (e.g., self-reflection) and with higher beings (e.g., communication with God). Thus, if chatbots become ubiquitous in religious contexts, the role of adherents might shift, as might the related symbolism.

Our study also sheds light on the work of third spaces [33] in the digital religion research. Existing as a hybrid space in between online and offline settings, third spaces embody what Hoover and Echchaibi [33] describe as an as-if-ness, i.e., people approach these spaces as if they are authentic [3]. Our interview data suggests that chatbots could engender third spaces that (re)define authenticity for their religious and spiritual practices. While this could bring trustworthy information related to the religious belief, expand access for others, and encourage them to reflect and grow spiritually, it could also engender a sense of inauthenticity around spiritual experiences due to the lack of human touch and the danger of substituting human effort.

Our work is also connected to the recent work by Natale on Deceitful Media [11]. This work essentially argues that communicative AI does not have its own intelligence but is developed according to the user's expectations for communication hence it reflects the user. The term "banal deception" is used to show that the intelligence is created such that it aligns with our existing social conventions and practices. From this perspective, our interviewees' feedback on how and what the chatbot should respond provides insights on their perspectives and understandings of how religious practices should be carried out. A next step from this 
direction is to examine people's understandings on the boundary of AI in digital religion - how intelligent can a machine be in this context and what are the aspects that the machine should not be intelligent about. In other words, our definitions and expectations of AI need to be clearly defined or recognized in the digital religion research.

Our study also makes methodological contributions. First, our multi-religion approach enables us to examine the design requirements of a spiritual chatbot in general. The design implications presented above are across different religions. Second, our visuals worked well in terms of engaging participants in speculative thinking processes about the use of intelligent technologies in religious activities. The screenshots generated additional interesting and insightful feedback that may otherwise be missed in the interviews.

A major limitation of our study is that the majority of our interviewees are regular members of their religious communities as opposed to leaders. Religious leaders use technologies to aid in their stewardship roles such as pastoral care [2]. We anticipate different intentions and perceptions for how they would perceive and/or use chatbots, particularly around how to maintain and exercise influence. Secondly, our participants are mainly university students who are more likely to be familiar with intelligent technologies, which are not representative of all community members. Finally, as the purpose of this exploratory study was to identify the main perceived benefits and challenges of using chatbot in religious activities as opposed to comparing and contrasting them among different religions, the sample we collected could not be used for a detailed religionspecific analysis or comparison, e.g., there were only four Buddhists and one Muslim in the sample. Future research efforts will gather more data and perform indepth analysis across and between different religions.

\section{Conclusion}

Despite the versatility and increasing popularity of chatbots, they have received little attention in digital religion research. As a step to close this gap, we explored the perceptions and preferences for chatbots in spiritual practices. We interviewed 23 people, comprising 12 Christians, 5 Hindus, 4 Buddhists, 1 Muslim, and 1 Pagan. Our findings show that participants have mixed feelings about using chatbots in their religious activities. On the positive side, a chatbot may be an intelligent information retrieval tool and a companion that prompts users to reflect on their emotions and spiritual activities. On the other side, it is concerning that chatbots may perform tasks that people are supposed to undertake due to their religious belief.
Additionally, there are and should be activities that chatbots cannot engage in because there should be a sacred communication space just between God and people. Finally, a chatbot cannot fulfil the need for human touch desired in these spaces.

We also developed a chatbot prototype using Juji Studio, an online platform that allows users to design, build, and launch the conversation flow and persona of a chatbot, all without coding. In our interviews, we presented the screenshots of a user interacting with the chatbot prototype for three envisioned conversation scenarios: asking the chatbot for religious information, engaging in a general spiritual conversation with the chatbot, and sharing a personal concern to the chatbot. The participants' feedback regarding these scenarios are both positive and skeptical. While they appreciate the chatbot's ability to provide valuable information to the user, they remain unsure about the domain knowledge a chatbot possesses to provide valuable advice to a user in need. Additionally, participants had mixed responses as to how human-like the chatbot should be in this context. Participants also offered design suggestions such as the type of questions a chatbot may ask and how.

\section{References}

[1] A. E. Grant, A. F. Sturgill, C. H. Chen and D. A. Stout eds., "Religion Online: How Digital Technology Is Changing the Way We Worship and Pray [2 volumes]", ABC-CLIO, 2019.

[2] S. P. Wyche, G. R. Hayes, L. D. Harvel and R. E. Grinter, "Technology in spiritual formation: an exploratory study of computer mediated religious communications", In Proceedings of the 2006 20th anniversary conference on Computer supported cooperative work (CSCW), pp. 199-208, 2006.

[3] H. A. Campbell and G. Evolvi, "Contextualizing current digital religion research on emerging technologies", Human Behavior \& Emerging Technologies, vol. 2, no. 1, pp. 5-17, 2020.

[4] M. Lövheim, "Religious socialization in a media age", Nordic Journal of Applied Ethics/Etikk i praksis, vol. 6, no. 2, 2012.

[5] S. P. Wyche, K. E. Caine, B. Davison, M. Arteaga and R. E. Grinter, "Sun dial: exploring techno-spiritual design through a mobile islamic call to prayer application", In CHI '08 Extended Abstracts on Human Factors in Computing Systems, pp. 3411-3416, 2008.

[6] W. Gaver, M. Blythe, A. Boucher, N. Jarvis, J. Bowers and P. Wright, "The prayer companion: openness and specificity, materiality and spirituality", In Proceedings of the SIGCHI conference on Human Factors in Computing Systems, pp. 2055-2064, 2010.

[7] X. L. Pham, T. Pham, Q. M. Nguyen, T. H. Nguyen and T. T. H. Cao, "Chatbot as an Intelligent Personal Assistant for Mobile Language Learning", In Proceedings of the 
$20182^{\text {nd }}$ International Conference on Education and ELearning, pp. 16-21, 2018.

[8] A. Xu, Z. Liu, Y. Guo, V. Sinha and R. Akkiraju, "A New Chatbot or Customer Service on Social Media", In Proceedings of the 2017 CHI Conference on Human Factors in Computing Systems, pp. 3506-3510, 2017.

[9] K. K. Fitzpatrick, A. Darcy and M. Vierhile, "Delivering cognitive behavior therapy to young adults with symptoms of depression and anxiety using a fully automated conversational agent (woebot): A randomized controlled trial”, JMIR Mental Health, vol. 4, no. 2, 2017.

[10] E. Buie, "Let us say what we mean: Towards operational definitions for techno-spirituality research", In Extended Abstracts of the 2019 CHI Conference on Human Factors in Computing Systems, pp. 1-10, 2019.

[11] Natale, S. (2021). Deceitful media: Artificial Intelligence and social life after the Turing Test. Oxford University Press, USA.

[12] M. Blondheim, E. Katz. "Religion, communications, and Judaism: the case of digital Chabad", Media, Culture \& Society, vol. 38, pp. 89-95, 2016. doi: $10.1177 / 0163443715615417$

[13] P. H. Cheong, A. Halavais, K. Kwon. "The Chronicles of Me: Understanding Blogging as a Religious Practice." Journal of Media and Religion, vol. 7, pp.107-131, 2008. doi:10.1080/15348420802223015

[14] D. Löffler, J.Hurtienne\& I. NordI. "Blessing Robot BlessU2: A Discursive Design Study to Understand the Implications of Social Robots in Religious Contexts". International Journal of Social Robotics, vol. 13, pp. 569586, 2021. https://doi.org/10.1007/s12369-019-00558-3

[15] Abu Shawar, BA and Atwell, ES (2004) An Arabic chatbot giving answers from the Qur'an.In: Bel, B and Marlien, I, (eds.) Proceedings of TALN04: XI Conference sur le Traitement Automatique des Langues Naturelles. TALN04: XI Conference sur le Traitement Automatique des Langues Naturelles, 19-22 April 2004, Fez, Morocco. ATALA , 197 - 202. ISBN 2-9518233-5-5

[16] M. T. Sihotang, I. Jaya, A. Hizriadi and S. M. Hardi, "Answering Islamic Questions with a Chatbot using Fuzzy String-Matching Algorithm", Journal of Physics: Conference Series, vol. 1566, no. 1, 2020.

[17] N. de Oliveira Castro, "Esperanza: Un chatbot diseñado para enseñar la Biblia por Facebook Messenger y WhatsApp", 2019 , http://repositorio.upeu.edu.pe/handle/UPEU/2314

[18] P. Pataranutaporn et al., "Buddha Bot: The Exploration of Embodied Spiritual Machine in Chatbot", In Proceedings of the Future Technologies Conference, pp. 589-595, 2019. Springer, Cham.

[19] E. B. N. Sanders and P. J. Stappers, "Co-creation and the new landscapes of design", CoDesign, vol. 4, no. 1, pp. 5$18,2008$.

[20] M. Deininger, S. R. Daly, J. C. Lee, C. M. Seifert and K. H. Sienko, "Prototyping for context: exploring stakeholder feedback based on prototype type, stakeholder group and question type", Research in engineering design, vol. 30, no. 4, pp. 453-471, 2019.

[21] G. R. Gibbs, "Thematic coding and categorizing", Analyzing qualitative data, no. 703, pp. 38-56, 2007.

[22] M. Moberg, S. Sjö, B. W. K. Golo, H. Erdiş Gökçe, R. F. Hart, S. C. Cardenas, F. Benyah and M. J. V. Jó, "From socialization to self-socialization? Exploring the role of digital media in the religious lives of young adults in Ghana, Turkey, and Peru", Religion, vol. 49, no. 2, pp. 240-261,2019.

[23] M. Klingenberg and S. Sjö, "Theorizing religious socialization: a critical assessment", Religion, vol. 49, no. 2, pp. 163-178, 2019.

[24] K. A. Puffer, K. G. Pence, T. M. Graverson, M. Wolfe, E. Pate and S. Clegg, "Religious doubt and identity formation: Salient predictors of adolescent religious doubt", Journal of Psychology and Theology, vol. 36, no. 4, pp. 270-284, 2008.

[25] A. M. Seeger, J. Pfeiffer and A. Heinzl, "When do we need a human? Anthropomorphic design and trustworthiness of conversational agents", In Proceedings of the Sixteenth Annual Pre-ICIS Workshop on HCI Research in MIS, AISeL, Seoul, Korea, vol. 15, 2017. http://aisel.aisnet.org/sighci2017/15

[26] A. P. Chaves and M. A. Gerosa, "How should my chatbot interact? A survey on social characteristics in human-chatbot interaction design", International Journal of Human-Computer Interaction, vol. 37, no. 8, pp. 729-758, 2020.

[27] A. Smith, "Cognitive empathy and emotional empathy in human behavior and evolution", The Psychological Record, vol. 56, no. 1, pp. 3-21, 2006.

[28] K. S. Nie, C. P. Kee and A. L. Ahmad, "Mediatization: A grand concept or contemporary approach?", Procedia-Social and Behavioral Sciences, vol. 155, pp. 362-367, 2014.

[29] D. M. Rousseau, S. B. Sitkin and R. S. Burt, "Not so different after all: A cross-discipline view of trust", Acad. Manag. Rev., vol. 23, no. 3, pp. 393-404, 1998.

[30] Z. Xiao, M. X. Zhou, W. Chen, H. Yang and C. Chi, "If I hear you correctly: Building and evaluating interview chatbots with active listening skills", In Proceedings of the 2020 CHI Conference on Human Factors in Computing Systems, pp. 1-14, 2020.

[31] H. A. Campbell (Ed.), "Digital religion: Understanding religious practice in new media worlds", Routledge, 2012.

[32] F. Krotz, "Media Connectivity: Concepts, Conditions, and Consequences", Network, Connectivity and Flow: Conceptualising Contemporary Communications, pp. 13-31, 2008.

[33] S. Hoover and N. Echchaibi, "The 'third spaces' of digital religion", The Center for Media, Religion, and Culture, 2012. 